\title{
Transformative changes in Distributed Acoustic Sensing (DAS) hydraulic fracture and VSP monitoring
}

David E. Diller*, NanoSeis, LLC, and Pete Richter, Silixa, LLC

Copyright 2019, SBGf - Sociedade Brasileira de Geofísica

This paper was prepared for presentation during the $16^{\text {th }}$ International Congress of the Brazilian Geophysical Society held in Rio de Janeiro, Brazil, 19-22 August 2019.

Contents of this paper were reviewed by the Technical Committee of the $16^{\text {th }}$ International Congress of the Brazilian Geophysical Society and do not necessarily represent any position of the SBGf, its officers or members. Electronic reproduction or storage of any part of this paper for commercial purposes without the written consent of the Brazilian Geophysical Society is prohibited.

\section{Abstract}

Two recent changes in Distributed Acoustic Sensing (DAS) hydraulic fracture and VSP monitoring are, in the authors' opinions, worthy of the term transformative. Firstly, engineered fibers provide an improvement in DAS acoustic sensitivity of $20 \mathrm{~dB}$ or more, and commonly allow detection on the order of thousands of microseismic events per stage in the Wolfcamp Formation of the Permian Basin, USA, and a typical listening distance of 1,000-1,300 meters depending on the definition of listening distance. Secondly, it is no longer necessary to go to the expense and risk of cementing fibers behind casing to get good quality DAS data. Fibers in wirelines that are simply lying inside the casing of a horizontal well, coupled only by gravity and friction, produce good data quality for both DAS acoustic waves and slow strain data. The deployment and use of temporary DAS fibers is far less expensive than that of permanent fibers.

\section{Introduction}

Understanding fracture geometry and estimating stimulated rock volume (SRV) is an important goal of operators in unconventional reservoirs. During hydraulic fracture treatment acoustic energy distribution and temperature profiling can be recorded in real time to understand which perforations are accepting fluid. A DAS fiber can also be used to acquire seismic and microseismic data at different stages of the well completion, and low frequency cross-well strain can be extracted from the data. The combined microseismic, cross-well strain, and temperature data are used to better understand rock properties, well interference, and for optimizing well spacing.

The utilization of distributed fiber measurements has been increasing over the past few years. We present here a Distributed Acoustic Sensing system that utilizes a new generation of engineered optical fiber with $20 \mathrm{~dB}$ or more of improved sensitivity compared to that of standard fibers. We also present results from temporary DAS fibers that are deployed in wirelines inside of casing.

\section{Method}

For DAS data each digital sample is indexed by the center location of a moving window along a cable's core (the sample's "channel", z) and recording time (the sample's time, $t)$. Thus if $u(z, t)$ represents the dynamic displacement of the fiber at axial location $z$ and time $t$, the DAS output is a measure of:

$$
\left[u\left(z+\frac{d z}{2}, t+d t\right)-u\left(z-\frac{d z}{2}, t+d t\right)\right]-\left[u\left(z+\frac{d z}{2}, t\right)-u\left(z-\frac{d z}{2}, t\right)\right]
$$

The traditional DAS systems utilize standard single mode fiber (Parker et al., 2014). However, a large improvement in the measurement sensitivity has been achieved by both advancing the state of the DAS optoelectronics interrogator architecture, together with the introduction of engineered fiber. This fiber is engineered with brighter backscatter along its length to reflect more laser light back to the interrogator, as shown in Figure 1 below. This is achieved without introducing significant loss in the forward-propagating laser pulses.

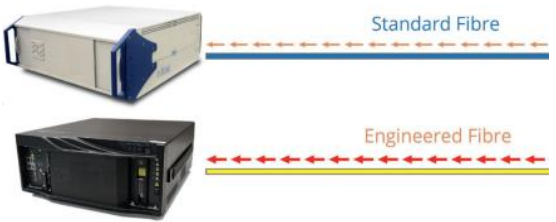

Figure 1. A cartoon depiction of how an engineered fiber reflects more laser light back to the interrogator than a standard fiber.

In addition to data improvements from engineered fiber, we have tested temporary fibers that are deployed inside wirelines that are lying inside the casing of horizontal or deviated wellbores, as shown in Figure 2 below.

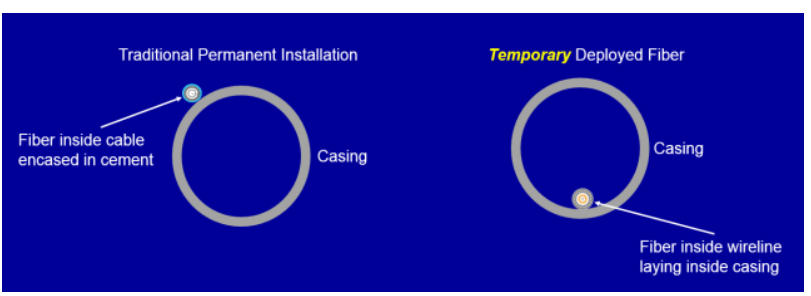

Figure 2. Cross-sectional view of a traditional permanently installed fiber that is cemented behind casing (left), and a temporary deployed fiber that is lying inside the casing (right).

\section{Examples and Results}

We first present examples and results that compare traditional DAS fibers with engineered fibers. Figure 3 below shows an example of the difference in signal/noise ratio for a microseismic event that was recorded with a traditional fiber DAS and an engineered DAS fiber, in the same location and in exactly the same way, except for the 
differences in the fibers and associated interrogators. The improvement in signal/noise from the engineered DAS fiber in this case is approximately $24 \mathrm{~dB}$.

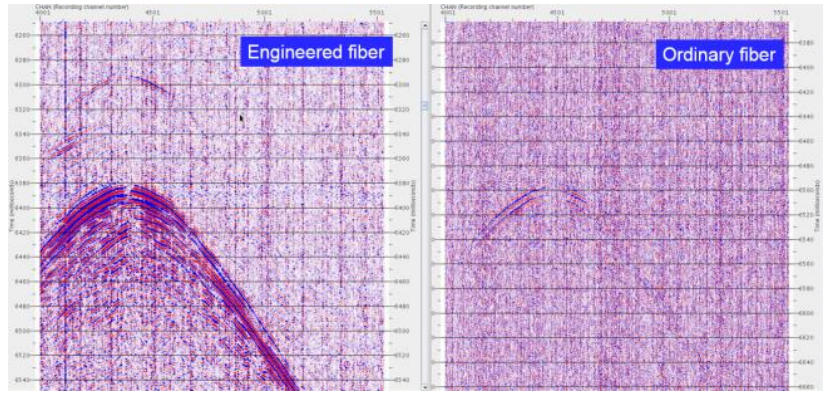

Figure 3. A single microseismic event recorded in the Permian Basin, USA, with an engineered DAS fiber (left) and the same microseismic event recorded with an ordinary DAS fiber in the same observation well (right). The displays are gained the same.

Microseismic results using traditional DAS data have been presented in the past (Webster et al., 2013), and while those results were promising the data quality was not comparable with downhole geophone data. We find that microseismic results using DAS data from engineered fibers are comparable, or perhaps better, than results using downhole geophone data. In our tests in the Permian Basin, USA, the effective listening distance was $1,000-1,300$ meters, where listening distance is defined as the distance from which energy from thousands of events travels far enough to be detected on the vertical part of the fiber, of where listening distance is defined as the distance from which the perforation shots can be detected by the fiber.

Figure 4 below shows an example of the data quality that is recorded using an engineered DAS fiber inside a temporary wireline, for a microseismic event in the Permian Basin, USA. In addition to the typically observed $\mathrm{P}$-wave and S-wave phases, reflected S-waves and an S$\mathrm{P}$ conversion are observed where the S-wave encountered vertical or nearly-vertical hydraulic fractures. Slow strain from the hydraulic fractures is evident as a bias in the trace amplitudes. The event that is shown was one of the largest events for that stage, but in that project almost every stage produced many events that showed S-wave reflections from the hydraulic fractures, which provides an intriguing opportunity to directly map the opening and closing behavior of the hydraulic fractures.

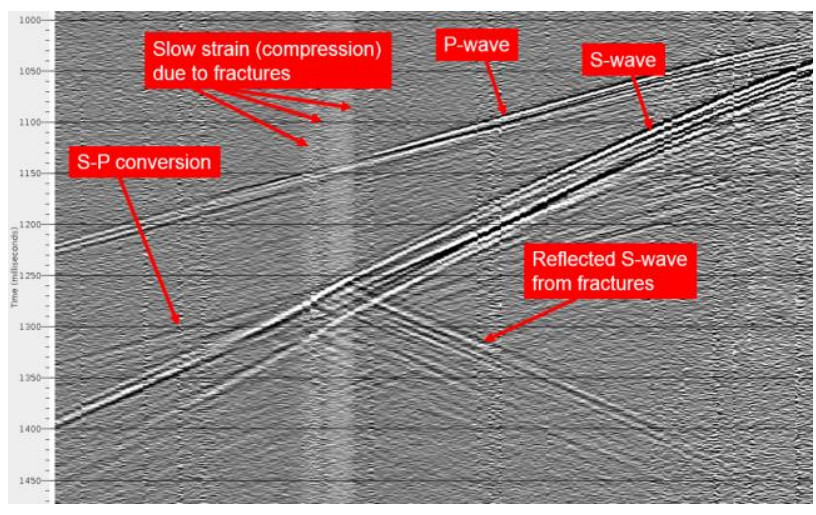

Figure 4. A microseismic event recorded in the Permian Basin, USA, with an engineered DAS fiber in a temporary wireline deployment.

Next we present examples and results that compare a permanent DAS fiber with a temporary DAS fiber. This is significant because temporary fibers can be tractored into wellbores or pumped down wellbores, which is far less expensive than cementing fibers behind the casing. Figure 5 below shows a microseismic event that was recorded in the same location and in exactly the same way, except that in one case the data was recorded with a permanently installed fiber that was cemented in place, and in the other case the data was recorded with a temporary fiber inside a wireline that is simply lying inside the casing. The data quality from the two fibers is very similar.
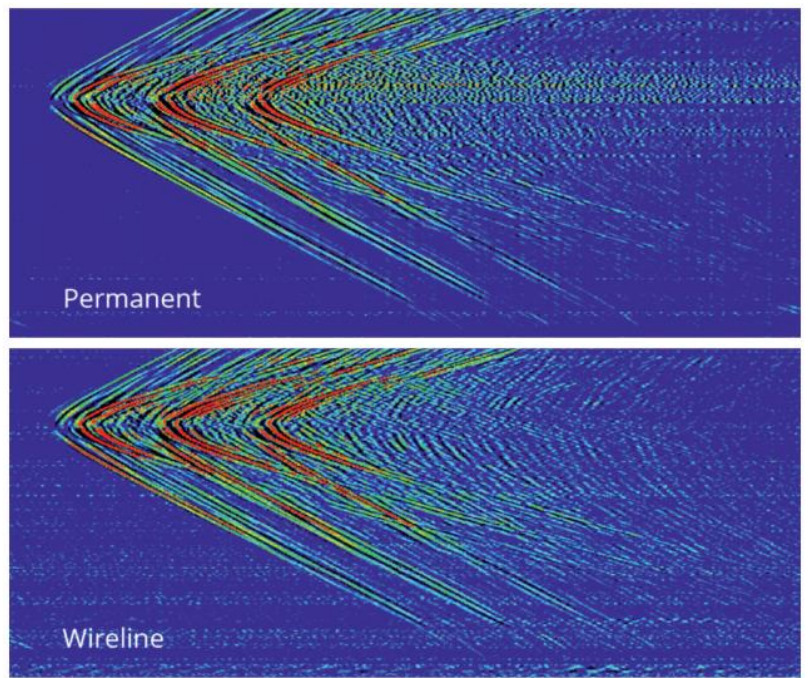

Figure 5. A single microseismic event recorded in the Permian Basin, USA, with a permanent fiber (upper) and a temporary fiber (lower) in the same observation well.

Slow strain that is extracted from DAS data provides a direct view of the rock deformation (Jin and Roy, 2017). Slow strain is typically presented in a "waterfall" plot as shown in Figure 6 below, in which the vertical axis is measured depth (or DAS channel) and the horizontal axis is time, typically measure in hours or days. Each pixel in a waterfall plot represents the 0 Hertz component of the data for one DAS channel for a short period of time, typically $15-30$ seconds.

While one might expect that acoustic waves would be detected by a temporary fiber, most people who had prior knowledge of the experiments did not expect that slow strain could be detected by a temporary fiber that is simply lying inside of the wellbore casing, because recording slow strain would require coupling between the casing and the wireline for relatively long periods of time (minutes), and the casing and wireline are only coupled by metal-on-metal friction with the aid of gravity. However, not only did the temporary fiber record the slow strain, the temporary fiber surprisingly appears to be more sensitive to slow strain than the permanent fiber, which is rather difficult to explain. 

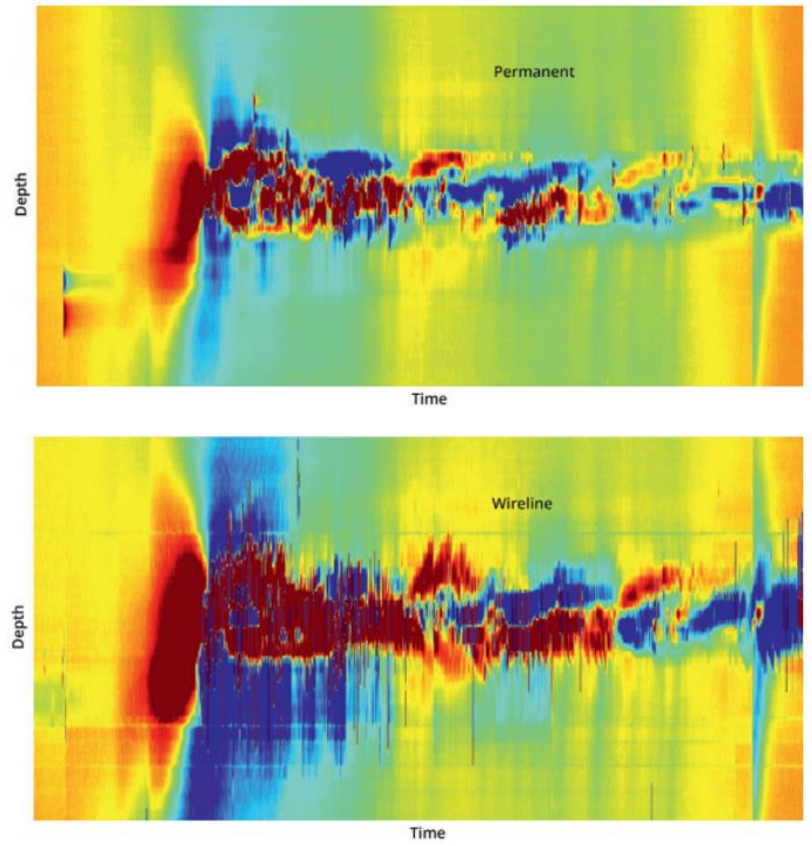

Figure 6. A slow strain anomaly recorded using a permanent fiber (above) and a temporary fiber (below). Both measurements are from the same locations as hydraulic fracture(s) approach and hit the horizontal observation wellbore.

Figure 7 below shows slow strain recorded with a temporary fiber in a horizontal well, showing the intersection of many fractures from three treatment wells during approximately 60 stages for approximately 10 days.

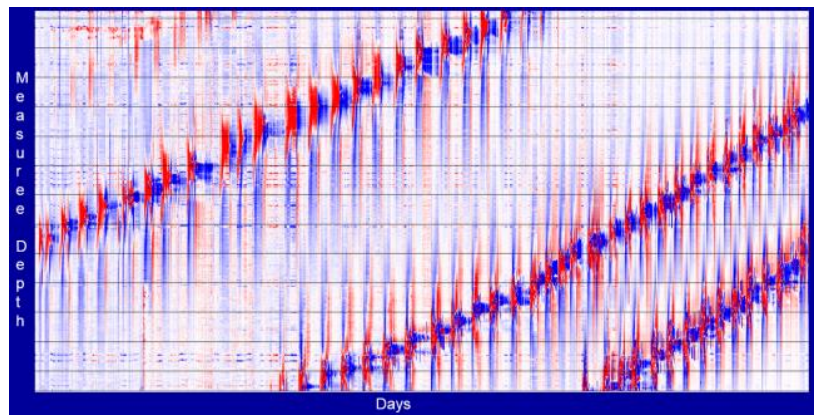

Figure 7. Slow strain anomalies recorded using a temporary fiber in a horizontal well for approximately 10 days.

Figure 8 below shows slow strain recorded using a temporary fiber in a horizontal well, showing the intersection of fractures during two stages. Many interesting features can be seen in the slow strain, including the strain signature of the approaching fractures, the intersection or "hits" of the fractures on the observation wellbore, and the strain signature of the closing fractures. Differences between the slow strain of two stages provide insights that can be used to optimize the number of perforation clusters and the stage spacing.

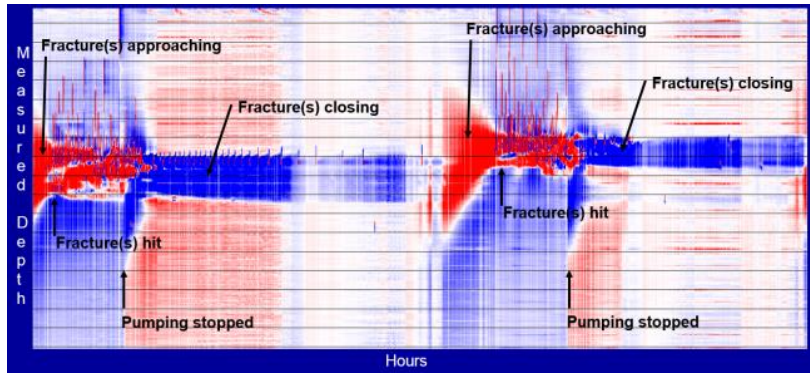

Figure 8. Slow strain anomalies recorded using a temporary fiber in a horizontal well, showing the approach, hitting, and closing of fractures during two stages.

Although most of the results presented here are for microseismic data, similar results are observed for VSP data. The VSP data quality from an engineered DAS fiber is much higher than the data quality from a traditional fiber. Also, the VSP data quality from a temporary DAS fiber is equal to or perhaps better than the data quality from a permanent fiber, as show in Figure 9 below. It should be noted that acquiring VSP data from a temporary fiber requires that the well be sufficiently nonvertical so that the wireline that contains the fiber is in contact with the casing.

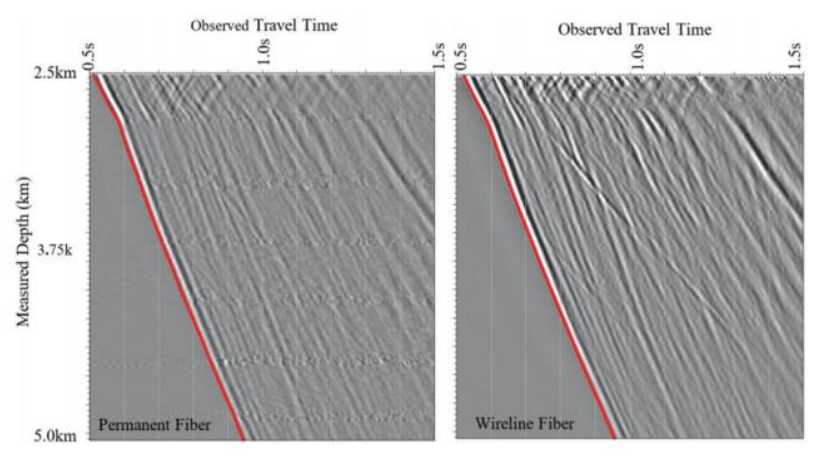

Figure 9. A VSP record recorded in the Permian Basin, USA, with a permanent fiber (left) and a temporary fiber (right) in the same deviated observation well.

\section{Conclusions}

We conclude that engineered DAS fibers provide an improvement in DAS acoustic sensitivity of $20 \mathrm{~dB}$ or more, and that good microseismic results and VSP results can be achieved with engineered DAS fibers. We futher conclude that DAS fibers in wirelines that are simply lying inside the casing of a horizontal well, coupled only by gravity and friction, produce good data quality for both DAS acoustic waves and slow strain data.

\section{Acknowledgments}

The authors are grateful to Silixa, LLC, for permission to show these results. 


\section{References}

Jin, G. and B. Roy [2017]. Hydraulic-fracture geometry characterization using low-frequency DAS signal. The Leading Edge, 975, 975-980.

Parker T. [2014]. Distributed Acoustic Sensing - a new tool for seismic applications. First Break, 32, 61-69.

Webster, P., B. Cox, and M. Molenaar [2013a]. Developments in diagnostic tools for hydraulic fracture geometry analysis. URTeC, 218-224, https:// doi.org/10.1190/urtec2013-025. 